\title{
A compensation index for multiattribute decision strategies
}

\author{
PIETER KOELE and MIRJAM R. M. WESTENBERG \\ University of Amsterdam, Amsterdam, The Netherlands
}

\begin{abstract}
In multiattribute decision problems, the subject has to evaluate a number of alternatives with given values on a number of attributes, in order to arrive at some conclusion about the attractiveness or utility of these alternatives. The information processing procedure leading to a conclusion is called a decision strategy, and one of the main research topics in multiattribute decision research has been the extent to which these strategies follow compensatory principles. Judges are said to follow compensatory strategies when low values on some attributes are compensated for by high values on other attributes. In process tracing studies using the information board technique, descriptions of decision strategies are usually based on three indices of the information search process: variability of search, search pattern (Payne, 1976), and depth of search. Variability of search, defined as the standard deviation of the proportion of information searched per alternative, is considered to give an indication of the degree of compensation of a decision strategy, compensation being smaller as variability increases. In this article, we propose an alternative way for establishing the degree of compensation of decision strategies in information board studies. We argue that the degree of compensation depends on both variability of search and depth of search (the proportion of information searched), and that a valid compensation index has to be a multiplicative function of these two indices.
\end{abstract}

In multiattribute decision problems, the subject has to evaluate a number of alternatives with given values on a number of attributes, in order to arrive at some conclusion about these alternatives, in terms of their utility, attractiveness, or suitability. The information processing procedure leading to a conclusion is called a decision strategy.

One of the frequently used methods for empirically identifying decision strategies is based on choice tasks using an information board. This board contains the information about all alternatives on all attributes in matrix format. Initially, this information is not visible. The subject has to search for this information by turning over cards, each card containing the value of one alternative on one attribute, or by clicking the mouse of a PC on a cell of the matrix, thus revealing the information.

One of the main issues in research on choice processes has been whether the decision strategy follows compensatory or noncompensatory principles (e.g., Westenberg and Koele, 1994). A strategy is said to be compensatory when low values on an attribute are compensated for by high values on other attributes. In other words, there is a tradeoff between the advantages and disadvantages of an alternative. Examples of compensatory strategies are the linear additive strategy and the additive difference strategy; examples of noncompensatory strategies are the conjunctive, the disjunctive, the lexicographic, and the

Correspondence should be addressed to either author, Faculty of Psychology, Roetersstraat 15, 1018 WB Amsterdam, The Netherlands (e-mail:ml_koele@macmail.psy.uva.nl, orkp_westenberg@macmail. psy.uva.nl). elimination by aspects strategies (for formulations and descriptions of these and other decision strategies, see Ford, Schmitt, Schechtman, Hults, \& Doherty, 1989; Svenson, 1979).

Because the degree of compensation appears to be such an important topic in this research area, the question arises how the degree of compensation of a decision strategy is actually being determined. It is the purpose of the present study to discuss the manner in which this issue is dealt with in the process tracing paradigm using the information board technique and to propose some ideas for improving upon the compensation index currently used.

\section{The Information Board}

In process tracing research using the information board a subject's decision strategy can be analyzed by calculating the values of several indices of the information search process: variability of search and search pattern, as developed by Payne (1976), and depth of search (Ford et al., 1989). Variability of search is the standard deviation of the proportions of cards turned over per alternative. A standard deviation of zero occurs when an equal amount of information is searched for each alternative and is considered to be indicative of a compensatory strategy. Standard deviations larger than zero indicate the use of noncompensatory strategies. Pattern of search has to do with the order in which cards are turned over. This can be done mainly per attribute, or mainly per alternative. Pattern is defined as the number of alternative-wise moves minus the number of attributewise moves, divided by the sum of these two numbers. 
A negative value indicates a mainly attribute-wise search pattern, a positive value a mainly alternative-wise search pattern. Finally, depth of search is defined as the proportion of cards turned over and consequently reflects the total amount of information searched for.

In information board studies, four specific decision strategies are of particular interest since each of them implies a specific search mode - that is, a combination of variability and pattern, as was suggested by Payne (1976): the linear strategy (variability zero, alternativewise pattern), the conjunctive strategy (variability greater than zero, alternative-wise pattern), the elimination-byaspects strategy (variability greater than zero, attributewise pattern), and the additive difference strategy (variability zero, attribute-wise pattern).

One must realize that there are more possible strategies than the four mentioned above and that different strategies may lead to similar values of pattern and variability. The step from the values of the information board indices to a formal description of the strategy is an inductive one: execution of a well-defined strategy will lead to deductive values of the indices, but any formal classification of a strategy on the basis of the indices is only a more or less plausible generalization.

Klayman (1983) introduced contingency measures, which intend to overcome this problem by assigning a number to an empirical strategy reflecting the extent to which the strategy corresponds to a specific formal strategy. Thus, these measures are able to differentiate between various compensatory and noncompensatory strategies. However, they do not measure the degree of compensation of an empirical strategy. As a matter of fact, they may be seen as measures of the goodness of fit of formal strategies on empirical strategies. Consequently, these measures have the disadvantage that they are dependent on the way these formal strategies are operationalized. Besides, given the large number of formal strategies for multiattribute decision making, the number of contingency measures describing a single empirical decision strategy may be equally large, which will seriously impair a straightforward interpretation of information board data. Nevertheless, we agree with Klayman that "strategies... differ along several continuous parameters" (Klayman, 1983, p. 410), and that is why we consider it to be important to develop a measure that represents one of the most important of these parameters (i.e., compensation).

Such a measure should be based on two aspects of the information search process-variability of search and the total amount of information searched, depth of search-because they are both indicative of compensatory strategies (Ford et al., 1989). When a subject turns over the cards for each alternative on only one attribute (not even necessarily the same one for each alternative), this subject receives a variability of zero but can hardly be considered to have followed a compensatory strategy. A small variability is necessary but is not a sufficient condition for a high degree of compensation. A strategy can be said to be compensatory only when the subject has searched a large amount of information and an approximately equal amount of information per alternative. We shall now introduce an index that integrates the information supplied by both variability and depth of search, yielding a continuous compensation index.

\section{A Compensation Index}

Compensatory decision strategies will lead to information search processes characterized by a relatively large amount of information processed and a more or less constant amount of information processed per alternative, because this is the only way in which a real tradeoff between attributes within an alternative can be made. In terms of the information board indices, compensatory strategies correspond to large values for depth of search and small values for variability of search. Because depth and variability determine the degree of compensation of a strategy jointly, a compensation index (denoted by $C$ from now on) must be a function of both of these indices. It is convenient to have $C$ range from zero to one, just like depth, with increasing values indicating more compensation. Before we can integrate the information supplied by depth and variability into the compensation index $C$, a transformation of variability must be executed that leads to transformed values ranging from zero to one, with increasing values indicating more compensation. After that, the precise function form relating $C$, depth, and variability can be defined.

The required transformation of variability is done in two steps. In the first step, the goal is to transform variability in such a way that it will range from zero to one. In the second step, the goal is to obtain a scale for this transformed variability on which zero indicates a fully noncompensatory strategy and one a fully compensatory strategy.

The first step involves dividing variability by its maximum value, $V_{\max }$. The value of $V_{\max }$ can be derived analytically as follows. Variability reaches its maximum value when half of the number of presented alternatives are inspected on all attributes, while the remaining alternatives are not inspected on any attribute. In other words, the proportions of cards turned over per alternative is one for half of the alternatives and zero for the other half. In this case, variability, the standard deviation of the proportions of cards turned over per alternative, is equivalent to the standard deviation of the distribution of a dichotomous variable (an "alternative distribution") with a proportion $p=.5$ ones and a proportion $1-p=.5$ zeros. This standard deviation of an alternative distribution equals $\sqrt{ }[p(1-p)]=\sqrt{ }[.5 \times .5]=.5$ (see, e.g., Johnson \& Kotz, 1969). So, the first step of the transformation is to divide variability $(V)$ by .5 , which is equivalent to multiplying it by a factor of 2 . As a result, this term $2 \times V$ ranges from zero to one, but with increasing values indicating less compensation. To have it the other way around, the second step of the transformation consists of taking the complement of the term 2 $\times V$, which results in $1-2 \times V$. $^{1}$ 
We now turn to the question of which function form should relate $C$ to depth and the transformation of variability. The most simple thing to do is to define $C$ as the mean of depth and transformed $V$, but this operation has the disadvantage that low values of one of the two indices can be (to a large extent) compensated for by high values of the other index. We want $C$ to be high only when both depth and transformed $V$ are high, and to be low when they are both low. Because depth and transformed $V$ both range from zero to one, this demand can be met by defining $C$ as the product of depth $(D)$ and transformed $V$ :

$$
C=D \times(1-2 V) .
$$

In the next section, we shall investigate some properties of $C$ as a measure of compensation of multiattribute decision strategies.

\section{Validity}

Before $C$ can be accepted as a valid compensation index, more has to be known about its psychometric and empirical properties. In this section, some results will be presented that give a first impression of some aspects of its construct validity (Cronbach, 1990). In particular, we calculated correlations between $C$ and depth and variability to get an impression of C's divergent validity, and we simulated a few decision strategies in an information board task in order to see how $C$ varies as a function of fundamental differences in degree of compensation of these strategies. As a product term, $C$ will tend to have substantial correlations with its factors depth and (a linear transformation of) variability. If these correlations are very high, $C$ has little divergent validity and will not add much to the interpretation of experimental results beyond the information already supplied by depth and variability.

In an unpublished study (Willems, Koele, \& Westenberg, 1995), we investigated (among others) the effect of two experimental manipulations on the information board measures: time pressure (high vs. low) and information load (high vs. low, by varying the number of alternatives). The results of this experiment will be reported elsewhere; what matters here are the correlations between $C$, depth, and variability. These correlations were calculated on the scores of 22 subjects (first-year psychology students at the University of Amsterdam) in each condition and had the following mean values: between $C$ and depth, .73 (ranging from .37 to .85 ); between $C$ and variability, -.72 (ranging from -.87 to -.40 ); between depth and variability, -.12 (ranging from -.45 to .34 ). These results show that, despite it being a product term, $C$ is not a fully redundant measure, because a considerable amount of its variance cannot be accounted for by either depth or variability.

We simulated a few decision strategies in order to demonstrate how $C$ varies. They are all two-phase strategies, with a noncompensatory strategy in the first, or screening, phase - intended to reject unsuitable alternatives - and a compensatory strategy in the second phase- aimed at selecting the best alternative. There is clear evidence that subjects use such phased strategies in complex multiattribute decision tasks (see Beach and Potter, 1992, and Stevenson, Busemeyer, \& Naylor, 1990, for overviews of empirical findings), and it is precisely for those kinds of mixed strategies that we think our measure can be useful. We constructed a 6 (alternatives $A$ up to $F$ ) $\times 6$ (attributes $X_{1}$ up to $X_{6}$ ) information board with randomly drawn numbers between 3 and 9 in the cells. Three two-phase strategies were defined and simulated on this board.

In all strategies, the first phase is conjunctive and the second phase is linear additive. The cards are turned over alternative-wise, and an alternative is rejected as soon as an attribute value is equal to or below a certain threshold. Of the remaining alternatives, the one with the highest mean value is selected. In Strategy 1 , the threshold is 5 ; in Strategy 2, it is 4; and in Strategy 3, it is 3. Clearly, Strategy 1 has the lowest degree of compensation, because attribute values 5 and lower cannot be compensated for by higher attribute values; Strategy 3 has the highest degree of compensation, because only the value 3 cannot be compensated for. The results of these strategies are presented in Figure 1 .

Shaded cells are the ones that would be opened by a hypothetical subject executing the defined strategy. Values of depth, variability, and $C$ were calculated on these data. Underlined alternatives are the ones ultimately selected according to the different strategies.

As expected, Strategy 1 leads to a rather low value of $C$, because the high threshold in the conjunctive screening phase of the strategy leads to a relatively limited amount of information searched, as well as a large variability across alternatives. Strategies 2 and 3 show that the lowering of the threshold leads to more information searched, less variability, and, thus, increasing values of $C$. It is interesting to note that the three strategies lead to different choice alternatives.

In a previous section, we discussed four one-phase strategies. The compensatory linear additive strategy and the additive difference strategy will lead to boards with all cards turned over, and consequently depth $=1$, variability $=0$, and $C=1$. The noncompensatory conjunctive strategy and the elimination-by-aspects strategy will lead to values of $C$ smaller than one, depending on the cut-off threshold used.

These examples serve to illustrate the validity of $C$ as a compensation index. Of course, we have demonstrated only the face validity of $C$. More elaborate and refined simulations are needed as a sound and convincing contribution to establishing its construct validity. What we have tried to show in this section is that $C$ is not completely redundant and differentiates satisfactorily among decision strategies varying in degree of compensation.

\section{Discussion}

As a continuous measure of compensation, $C$ reflects the idea already formerly expressed by, for instance, 
Attribute

\begin{tabular}{|c|c|c|c|c|c|c|}
\hline & $\mathrm{X}_{1}$ & $\mathrm{X}_{2}$ & $\mathrm{X}_{3}$ & $\mathrm{X}_{4}$ & $\mathrm{X}_{5}$ & $\mathrm{X}_{6}$ \\
\hline A & 9 & 7 & 5 & 6 & 7 & 7 \\
\hline B & 6 & 7 & 8 & 3 & 5 & 4 \\
\hline C & 9 & 4 & 5 & 8 & 7 & 9 \\
\hline D & 7 & 7 & 6 & 7 & 6 & 6 \\
\hline E & 6 & 5 & 5 & 6 & 5 & 6 \\
\hline F & 6 & 6 & 7 & 6 & 7 & 8 \\
\hline
\end{tabular}

Strategy 1 . Consider each alternative on the attributes, starting with attribute $X_{1}$. Reject an alternative as soon as it has an attribute value of 5 or lower. Of the remaining alternatives, select the one with the highest mean attribute value.

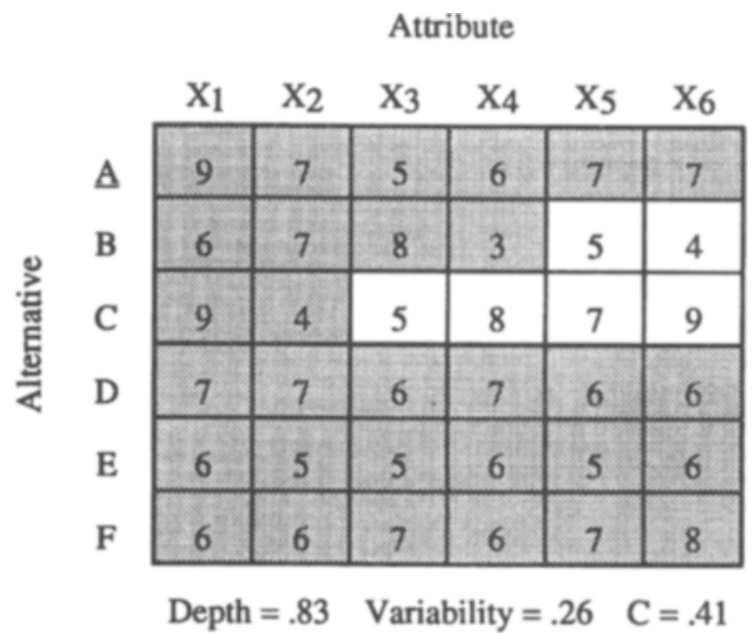

Strategy 2. Consider each alternative on the attributes, starting with attribute $X_{1}$. Reject an alternative as soon as it has an attribute value of 4 or lower. Of the remaining alternatives, select the one with the highest mean attribute value.

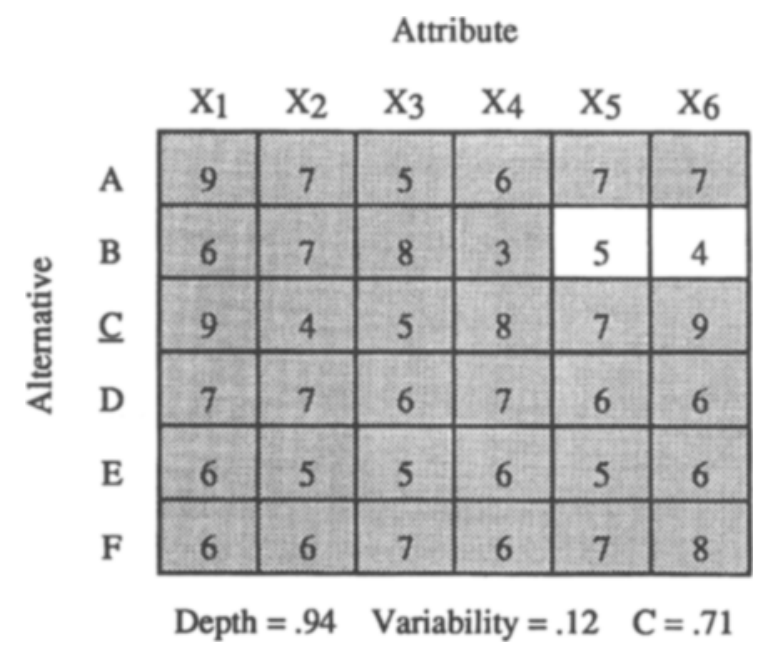

Strategy 3. Consider each alternative on the attributes, starting with attribute $X_{1}$. Reject an alternative as soon as it has an attribute value of 3 or lower. Of the remaining alternatives, select the one with the highest mean attribute value.

Figure 1. Examples of multiattribute decision strategies and corresponding values of information board measures.

Klayman (1983) and Ford et al. (1989) that empirical decision processes are not either this or that but rather possess certain properties to a lesser or greater extent. As such, $C$ may serve a useful purpose as a dependent variable in experimental designs or as a predictor or criterion variable in correlational studies. In the former case, $C$ can be used to assess differences in the degree of compensation of decision strategies as a function of experimental manipulations. In the latter case, individual differences in the degree of compensation of decision strategies can be related to individual differences on psychological, physiological, or behavioral variables.
We consider this psychometric approach to the issue of the degree of compensation of decision processes to be urgently needed. In our opinion, it makes little sense to assign labels of formal models to empirical decision strategies. First, the inductive leap from the values of the information board measures to these models is rather arbitrary. Second, and most important, we feel that it is time to make a serious attempt to explain individual differences with respect to the degree of compensation of decision strategies from a theoretic psychological point of view. For that purpose, a reliable and valid measure of compensation as a personal characteristic is necessary. 
We hope that the introduction of our measure $C$ may serve as a stimulating development into that direction.

\section{REFERENCES}

BEACH, L. R., \& PotTER, R. E. (1992). The pre-choice screening of options. Acta Psychologica, 81, 115-126.

Cronbach, L. J. (1990). Essentials of psychological testing (5th ed.) New York: Harper \& Row.

Ford, J. K., SChmitt, N., Schechtman, S. L., Hults, B. M., \& DoHERTY, M. L. (1989). Process tracing methods: Contributions, problems, and neglected research issues. Organizational Behavior \& Human Decision Processes, 43, 75-117.

Johnson, N. L., \& Kotz, S. (1969). Discrete distributions. New York: Wiley.

KLAYMAN, J. (1983). Analysis of pre decisional information search patterns. In P. C. Humphreys, O. Svenson, \& A. Vari (Eds.), Analysing and aiding decision processes (pp. 401-414). Amsterdam: Elsevier, North-Holland.

PAYNE, J. W. (1976). Task complexity and contingent processing in decision making: An information search and protocol analysis. Organizational Behavior \& Human Performance, 16, 366-387.

Stevenson, M. K., Busemeyer, J. R., \& NAYlor, J. C. (1990). Judgment and decision-making theory. In M. D. Dunette \& L. M. Hough (Eds.), Handbook of industrial and organizational psychology (Vol. 1, pp. 283-374). Palo Alto: Consulting Psychologists Press. SVEnson, O. (1979). Process descriptions of decision making. Organizational Behavior \& Human Performance, 23, 86-112.
Westenberg, M. R. M., \& Koele, P. (1994). Multiattribute evaluation processes: Methodological and conceptual issues. Acta Psychologica, 87, 65-84.

WILlEMs, P., Koele, P., \& Westenderg, M. R. M. (1995). A comparison of different information board techniques. Unpublished manuscript, University of Amsterdam, Faculty of Psychology.

\section{NOTE}

1. In the transformation, thus far, it was assumed that the number of alternatives $n$ is even. In case of an odd number $n$ of alternatives, variability reaches its maximum value if there are $.5(n-1)$ ones and $.5(n+1)$ zeros, or vice versa. Then the proportion of ones equals $p=$ $.5(n-1) / n$, and the proportion of zeros equals $1-p=.5(n+1) / n$, and consequently $V_{\max }=\sqrt{[p(1-p)]=}$

$$
V_{\max }=\sqrt{\frac{5(n-1) \times .5(n+1)}{n^{2}}}=.5 \sqrt{\frac{n^{2}-1}{n^{2}}} .
$$

When $n$ approaches infinity, the term under the radical sign approaches 1 , and $V_{\max }$ approaches .5 . In reality, this limit is reached very soon. For $n=3, V_{\max }$ equals .47; for $n=5$, it is .49 ; and from $n=7$ on, it equals .50. So for all practical purposes, it seems justified to take .5 as the maximum value of $V$, and consequently $1-2 \times V$ as its transformation.

(Manuscript received November 10, 1993; revision accepted for publication January 13,1995.) 